\title{
Overexpression of high mobility group box 1 and 2 is associated with the progression and angiogenesis of human bladder carcinoma
}

\author{
WEI WANG ${ }^{1}$, HAOWEN JIANG ${ }^{1,2}$, HECHEN ZHU ${ }^{3}$, HU ZHANG ${ }^{2}$, \\ JIAN GONG ${ }^{1}$, LIMIN ZHANG ${ }^{2}$ and QIANG DING ${ }^{1,2}$ \\ ${ }^{1}$ Institute of Urology, Departments of ${ }^{2}$ Urology and ${ }^{3}$ ICU, Huashan Hospital, Fudan University, Shanghai 200040, P.R. China
}

Received September 26, 2012; Accepted December 18, 2012

DOI: $10.3892 / \mathrm{ol} .2012 .1091$

\begin{abstract}
High mobility group box 1 (HMGB1) and HMGB2 overexpression has been observed in several human tumor types, and is involved in cancer progression and prognosis. However, the clinicopathological significance of HMGB1 and HMGB2 expression in bladder carcinoma (BCa), particularly the involvement of these proteins in angiogenesis, remains unclear. In the present study, immunohistochemistry and real-time polymerase chain reaction (PCR) of HMGB1 and HMGB2 in $64 \mathrm{BCa}$ patients revealed that HMGB1 and HMGB2 were overexpressed in BCa tissues compared with normal tissues, and were correlated with tumor clinical stage and pathological grade. In addition, correlation analysis of vascular endothelial growth factor (VEGF) and microvessel density (MVD) counts indicated that the overexpression of HMGB1 and HMGB2 was also correlated with angiogenesis. We conclude that HMGB proteins act as key regulators in the progression and angiogenesis of bladder carcinoma, and serve as potential diagnostic and therapeutic targets.
\end{abstract}

\section{Introduction}

Bladder carcinoma (BCa) is the most common genitourinary malignancy worldwide. The disease has been annually diagnosed in 55,600 males and 17,910 females in the USA and is the fourth most common type of cancer in males and the eighth most common in females (1). Fifty to seventy percent of initially diagnosed $\mathrm{BCa}$ patients experience a recurrence within 5 years; however, $10 \%$ progress to invasive $\mathrm{BCa}$ (2). The diverse biological behavior of $\mathrm{BCa}$ has forced current guidelines to recommend intense follow-up and invasive treatment, as no reliable method to determine the recurrence and invasive potential of such tumors has been identified (3). It is

Correspondence to: Dr Haowen Jiang, Department of Urology, Huashan Hospital, Fudan University, No. 12, WuLuMuQi Middle Road, Shanghai 200040, P.R. China

E-mail: drjianghaowen@126.com

Key words: high mobility group box, bladder carcinoma, angiogenesis important to predict the invasive behavior of $\mathrm{BCa}$ in clinical studies, and specific molecular markers that may serve as credible potential prognostic factors are required.

High mobility group box (HMGB) proteins are ubiquitous, abundant nuclear proteins with diverse functions in the cell. The proteins were first purified from nuclei in the 1970s and termed 'high mobility group' (HMG) proteins to reflect their rapid mobility in sodium dodecyl sulfate-polyacrylamide gel electrophoresis (SDS-PAGE) gels (4,5). HMGB1 and HMGB2 are the main members of the HMGB protein family. Unlike HMGB1, HMGB2 is highly expressed during embryogenesis, but has limited expression in adult organs, mainly in the lymphoid organs and testes. HMGB1 and HMGB2 are highly conserved with $>80 \%$ amino acid identity and similar molecular structures; both proteins have a tripartite domain organization, consisting of two DNA-binding domains, the HMG-boxes A and B, and acidic C-terminal tails of variable length $(6,7)$. HMGB1 and HMGB2 have indistinguishable biological properties within the nucleus, such as binding to DNA without sequence specificity and regulating transcription, replication, DNA repair and recombination (8-10). HMGB1 and HMGB2 are important in growth and development. An early study demonstrated that HMGB1 knockout mice did not survive within $24 \mathrm{~h}$ after birth, due to hypoglycemia, and they exhibited a defect in the transcriptional function of the glucocorticoid receptor. Additionally, the HMGB2 knockout mice survived into adulthood, although spermatogenesis was impaired (6).

In cancer chemotherapy, HMGB1 and HMGB2 may act as sensors of DNA modification and facilitate p53 phosphorylation following exposure to genotoxic stress, thus becoming newly identified components of the DNA damage signaling cascade and providing novel promising targets for chemotherapeutic intervention (7,11). HMGB1 has also been demonstrated to be released into the extracellular medium and to exhibit important effects in the mediation of tumor growth, angiogenesis and metastasis $(12,13)$. Overexpression of HMGB1 and HMGB2 has been observed in several human cancer types, including hepatocellular (14), skin squamous cell (15), prostate (16), gastrointestinal $(12,17)$ and breast carcinomas $(18,19)$. HMGB1 has been identified to be a latent pro-angiogenic factor in cancer progression and angiogenesis; it has been observed to initiate the production of angiogenic factors, such as vascular endothe- 
Table I. Correlation between HMGB1 or HMGB2 protein expression and clinicopathological features of the 64 patients with BCa.

\begin{tabular}{|c|c|c|c|c|c|}
\hline Parameter & Total & High HMGB1 expression & $\mathrm{P}$-value & High HMGB2 expression & P-value \\
\hline Gender & & & 0.637 & & 0.154 \\
\hline Female & 18 & 9 & & 3 & \\
\hline Male & 46 & 26 & & 16 & \\
\hline Age (years) & & & 0.451 & & 0.784 \\
\hline$\leq 65$ & 32 & 16 & & 10 & \\
\hline$>65$ & 32 & 19 & & 9 & \\
\hline T stage & & & 0.002 & & 0.010 \\
\hline Ta-T1 & 42 & 17 & & 8 & \\
\hline $\mathrm{T} 2-\mathrm{T} 4$ & 22 & 18 & & 11 & \\
\hline Pathological grade & & & $<0.001$ & & 0.016 \\
\hline Low & 35 & 12 & & 6 & \\
\hline High & 29 & 23 & & 13 & \\
\hline
\end{tabular}

H]MGB, high mobility group box; BCa, bladder carcinoma.

lial growth factor (VEGF) $(20,21)$. As a surrogate marker for angiogenesis, microvessel density (MVD) has been demonstrated to be predictive of progression and a poor prognosis of $\mathrm{BCa}(22,23)$. Although there has been extensive characterization of the various roles of HMGB1 in cancer, considerably less is known regarding the involvement of HMGB2 in carcinogenesis, including its angiogenic effects and precise signaling pathways.

The present study investigated the clinicopathological significance of HMGB1 and HMGB2 expression in human $\mathrm{BCa}$ by quantitative real-time polymerase chain reaction (PCR) and immunohistochemistry. Furthermore, the correlations between the expression of HMGB1/HMGB2 and VEGF as well as MVD counts have been analyzed, to explore the angiogenic role of HMGB1 and HMGB2 in BCa.

\section{Materials and methods}

Patients and tissue samples. Tumor tissue samples were collected from 64 patients with $\mathrm{BCa}$ and 15 normal bladder tissue samples were obtained from the Department of Urology, Huashan Hospital between January 2010 and July 2011. All tissues were confirmed by histological examination of sequential sections. Tumor staging was determined according to the sixth edition of the tumor node metastasis (TNM) classification of the International Union Against Cancer. Clinical information regarding the samples is described in detail in Table I. Ethical approval was obtained from the research ethics committee of Huashan Hospital, and written informed consent was obtained from all patients.

Real-time PCR. Total RNA was isolated from the tumor and normal tissues using TRIzol reagent (Invitrogen Life Technologies; Carlsbad, CA, USA). Total RNA ( $2 \mu \mathrm{g})$ was reverse transcribed to cDNA using M-MLV reverse transcriptase (Promega Corp., Madison, WI, USA). A typical $25 \mu 1$ reaction mixture contained $12.5 \mu 12 \mathrm{X}$ One Step SYBR RT-PCR buffer (Takara Bio. Inc., Shiga, Japan), $11 \mu \mathrm{l}$ of water, $0.5 \mu \mathrm{l}$ of template and $1 \mu \mathrm{l}$ of specific primers. The primer sequences were as follows: forward: 5'-ATATGGCAAAAGCGGA CAAG-3' and reverse: 5'-GCAACATCACCAATGGACAG-3' for HMGB1; forward: 5'-CGTTCCTCCCAAAGGTGATA-3' and reverse: 5'-TCTTTGGCTGACTGCTCAGA-3' for HMGB2; forward: 5'-GGCGGCACCACCATGTACCCT-3' and reverse: 5'-AGGGGCCGGACTCGTCATACT-3' for $\beta$-actin. An initial denaturation/activation step $\left(15 \mathrm{sec}\right.$ at $\left.95^{\circ} \mathrm{C}\right)$ was followed by 40 cycles $\left(5 \mathrm{sec}\right.$ at $95^{\circ} \mathrm{C}$ and $30 \mathrm{sec}$ at $\left.60^{\circ} \mathrm{C}\right)$. The relative expression of HMGB1 and HMGB2 mRNA was calculated with the $2^{-\Delta \Delta \mathrm{Ct}}$ method and normalized using the $\beta$-actin mRNA expression level. All experiments were performed in triplicate.

Immunohistochemistry and evaluation. Formalin-fixed and paraffin-embedded tissue sections $(5 \mathrm{~mm})$ were dewaxed with xylene and rehydrated through an ethanol gradient into water. Following blocking of endogenous peroxidase activity with $0.3 \%$ hydrogen peroxide for $10 \mathrm{~min}$, the sections were washed with phosphate-buffered saline (PBS) and incubated overnight with rabbit anti-HMGB1 antibody (ab92310; Abcam; Cambridge, MA, USA) or HMGB2 antibody (ab11973; Abcam), VEGF antibody (ab1316; Abcam) or CD34 antibody (ab81289; Abcam) at the dilution of 1:100 in a humidified chamber at at $4^{\circ} \mathrm{C}$. After washing with PBS, sections were incubated with biotinylated secondary antibody for $30 \mathrm{~min}$ at $37^{\circ} \mathrm{C}$ and then with horseradish peroxidase-labeled streptavidin for $30 \mathrm{~min}$ at $37^{\circ} \mathrm{C}$. Diaminobenzidine (DAB) was used as chromogen and the sections were subsequently counterstained with hematoxylin, then dehydrated, cleared and mounted.

Sections were evaluated and scored as described previously by Yang et al (24); the extent of staining was scored as $0(0 \%), 1(1-25 \%), 2(26-50 \%), 3(51-75 \%)$ or $4(76-100 \%)$, according to the percentage of positively stained areas. The staining intensity was scored as 0 (negative), 1 (weak), 2 (medium) or 3 (strong). The first and second scores were then added together to produce the final score (0-7) for HMGB1, HMGB2 or VEGF. Tumors with a final staining score of $>3$ were considered to have high protein expression and $<2$ were considered to have low protein expression. 

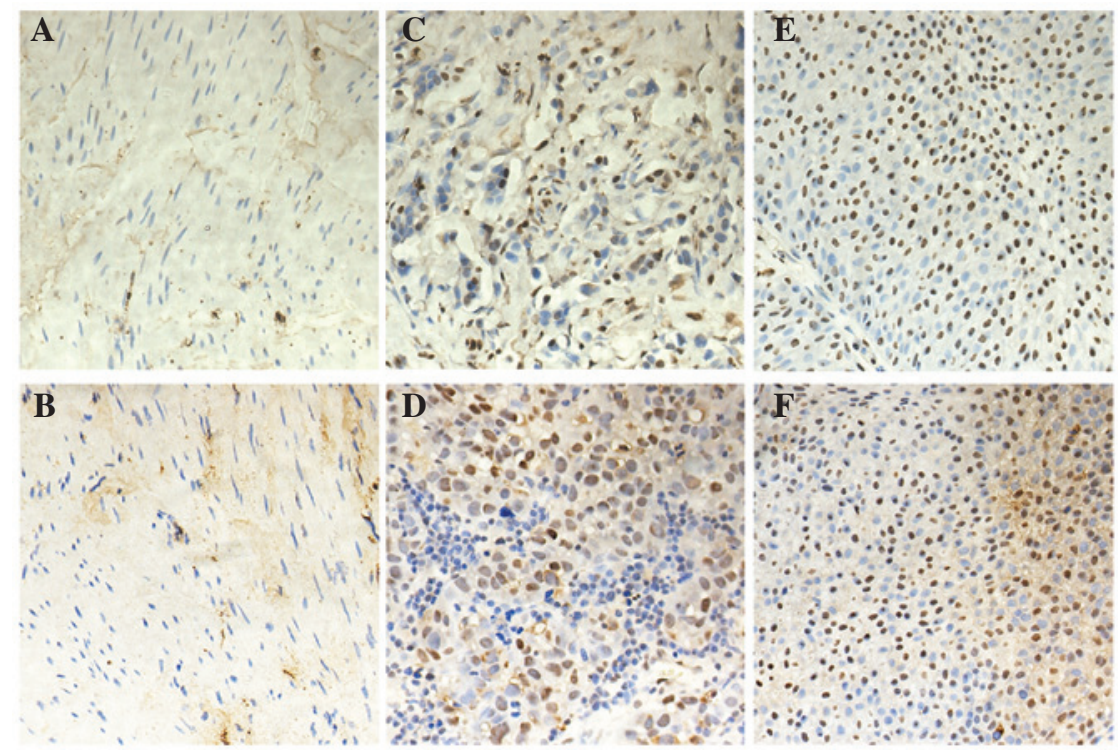

Figure 1. Immunohistochemical analysis of high mobility group box 1 (HMGB1) and HMGB2 expression in bladder carcinoma (BCa). HMGB1 (A) and HMGB2 (B) staining is negative in the majority of normal bladder urothelia. Low expression of HMGB1 (C) and HMGB2 (D) is evident in certain BCa Extensive expression of HMGB1 (E) and HMGB2 (F) is shown in BCa. Original magnification, $\mathrm{x} 200$.

A

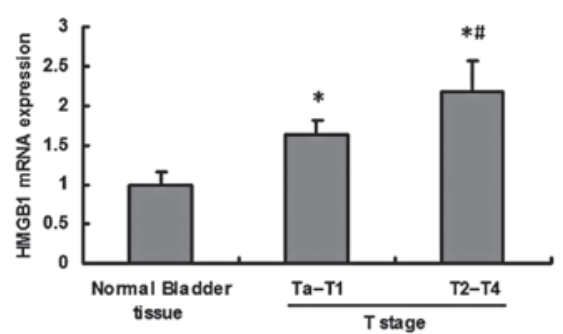

C

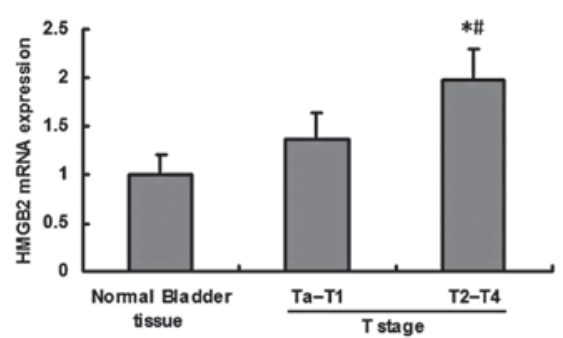

B

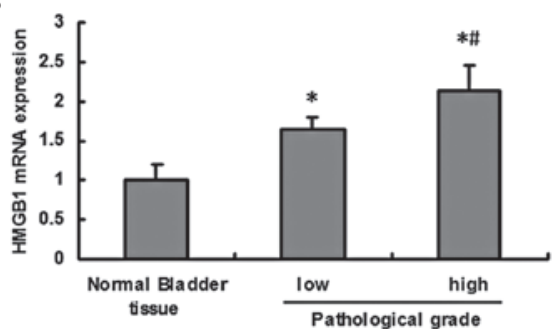

D

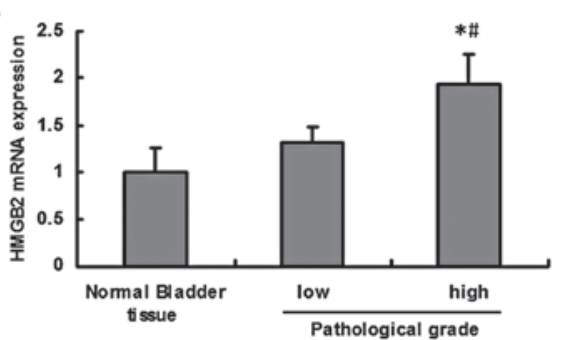

Figure 2. Real-time polymerase chain reaction (PCR) analysis of high mobility group box 1 (HMGB1) and HMGB2 mRNA expression in bladder carcinoma (BCa). (A) HMGB1 mRNA expression in BCa with different tumor (T) stages. (B) HMGB1 mRNA expression in BCa with different pathological grades. (C) HMGB2 mRNA expression in BCa with different T stages. (D) HMGB2 mRNA expression in BCa with different pathological grades. "P<0.05 vs. normal bladder urothelium, ${ }^{\#} \mathrm{P}<0.05$ vs. Ta-T1 stage or low pathological grade.
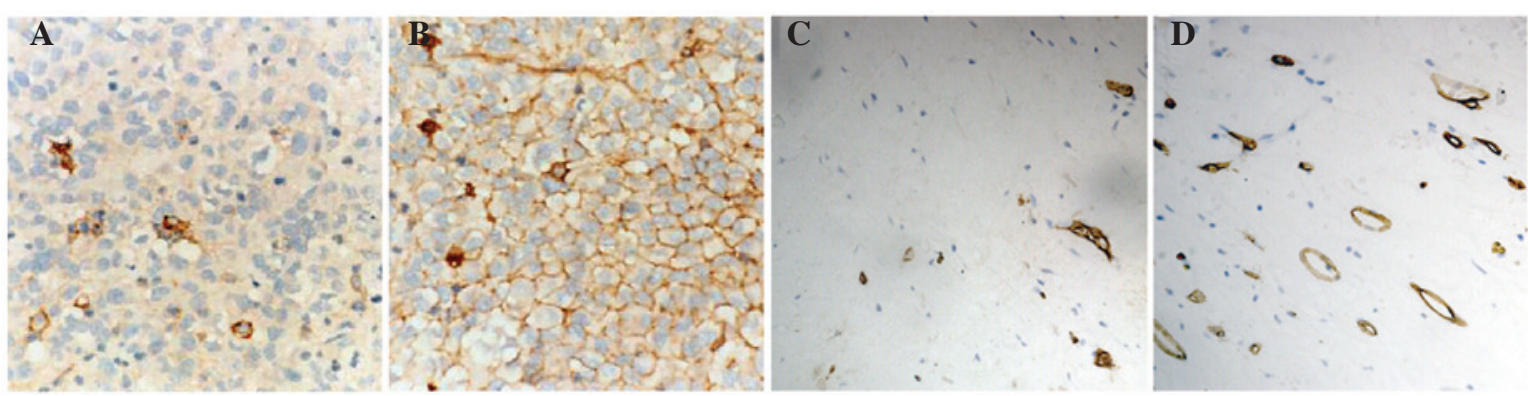

Figure 3. Immunohistochemical analysis of vascular endothelial growth factor (VEGF) and CD34 expression in bladder carcinoma (BCa). Low (A) and high (B) expression of VEGF in BCa. Low (C) and high (D) expression of CD34 in BCa. Original magnification, x200. 
Table II. Correlation between HMGB1 or HMGB2 protein expression and VEGF expression or MVD.

\begin{tabular}{|c|c|c|c|c|c|c|}
\hline Parameter & Total & Low VEGF expression & High VEGF expression & P-value & MVD & $\mathrm{P}$-value \\
\hline HMGB1 & & & & $<0.001$ & & $<0.001$ \\
\hline Low expression & 29 & 19 & 10 & & $23.0 \pm 6.4$ & \\
\hline High expression & 35 & 7 & 28 & & $35.5 \pm 10.8$ & \\
\hline HMGB2 & & & & 0.038 & & $<0.001$ \\
\hline Low expression & 45 & 22 & 23 & & $26.6 \pm 9.6$ & \\
\hline High expression & 19 & 4 & 15 & & $37.6 \pm 10.3$ & \\
\hline
\end{tabular}

HMGB, high mobility group box; VEGF, vascular endothelial growth factor; MVD, microvessel density.

CD34-stained whole sections were used to evaluate MVD as described previously by Ajili et al and Deniz et al $(22,23)$. Microvessel counts of five areas with the most intense neovascularization were performed at a magnification of $\mathrm{x} 200$.

Statistical analysis. The $\chi^{2}$ test was used to analyze differences in categorical variables. Statistical analyses were performed using a Student's two-tailed unpaired t-test for comparisons between two groups. All computations were performed with the Statistical Package for the Social Sciences (SPSS) 12.0 software (SPSS, Inc.; Chicago, IL, USA). P<0.05 was considered to indicate a statistically significant difference.

\section{Results}

HMGB1 and HMGB2 are overexpressed in BCa. Immunohistochemical analysis demonstrated that HMGB1 and HMGB2 were negative in the majority of normal bladder urothelia, but were positive in BCa (Fig. 1). A total of $35(55 \%)$ cases and $19(30 \%)$ cases had high expression of HMGB1 and HMGB2 protein, respectively (Table I). The correlation between HMGB1 and HMGB2 protein expression and the clinicopathological features of $\mathrm{BCa}$ demonstrated that levels of both HMGB1 and HMGB2 protein expression were significantly correlated with tumor grade and stage (Table I), and were not correlated with the remaining clinicopathological features tested, including gender and age.

Upregulation of HMGB1 and HMGB2 mRNA in BCa. Real-time PCR analysis of HMGB1 mRNA expression revealed that the mRNA expression of HMGB1 was significantly upregulated in $\mathrm{BCa}$, and the mRNA expression in T2-T4 stage or high pathological grade tumor tissue was significantly higher than that in Ta-T1 stage or low pathological grade tumor tissue (Fig. 2A and B). The mRNA expression of HMGB2 was significantly upregulated in T2-T4 stage or high pathological grade tumor tissue compared with normal bladder tissue and Ta-T1 stage or low pathological grade tumor tissue; whereas no marked differences were observed between normal bladder tissue and low grade tumor tissue (Fig. 2C and D).

Correlation between HMGB1 and HMGB2 protein expression and angiogenesis. To investigate angiogenesis in $\mathrm{BCa}$, immunohistochemistry of VEGF and CD34 was performed. As demonstrated in Fig. 3 and Table II, levels of VEGF protein expression and MVD were significantly correlated with levels of HMGB1 and HMGB2 protein expression.

\section{Discussion}

Our study found that HMGB1 and HMGB2 were overexpressed in $\mathrm{BCa}$ tissues compared with normal tissues, and were correlated with both the clinical stage and pathological grade of the tumor. In addition, overexpression of HMGB1/HMGB2 was also correlated with VEGF expression and MVD counts. This implies that HMGB1 and HMGB2 are likely to have key roles in the progression of $\mathrm{BCa}$.

Overexpression of HMGB1 has been observed in several human tumor types. HMGB1 may be involved in cancer progression and prognosis, including apoptosis, angiogenesis, the inflammatory microenvironment, mobility, invasion, metastasis and patient survival $(9,20)$. During cancer progression, HMGB1 has been observed to modulate the NF- $\mathrm{B}$, $\mathrm{PI} 3 \mathrm{~K} / \mathrm{AKT}$ and mitogen-activated protein kinase (MAPK) signaling pathways by interacting with the receptor for advanced glycation end products (RAGE), while blockade of the HMGB1/RAGE interaction has been demonstrated to suppress tumor growth and metastasis $(25,26)$. HMGB2 is highly homologous to HMGB1 and may exhibit similar effects in neoplastic development (27). Although HMGB1 has been studied more extensively, considerably less is known regarding HMGB2 in the study of cancer, particularly its relevance in carcinogenesis. To our knowledge, overexpression of HMGB2 has been identified in several types of tumors, including hepatocellular carcinoma (HCC) and skin cancer, and has been involved in cancer progression and prognosis via interacting with RAGE, steroid receptors, p53 and p73 (14,15). In addition, HMGB2 has been demonstrated to be significantly downregulated by the anti-human epidermal growth factor receptor 2 antibody through the AKT pathway in breast cancer cell lines (28). The present study indicated that HMGB1 and HMGB2 genes and proteins were overexpressed in BCa, using real-time PCR and immunohistochemical examination. The overexpression of HMGB/HMGB2 was correlated with both the clinical stage and pathological grade of the tumor, and HMGB1 and HMGB2 may be involved in BCa development and progression. Their involvement in the regulation of the carcinogenesis of $\mathrm{BCa}$ requires further investigation.

HMGB1 is a latent pro-angiogenic factor that has been observed to act both directly and indirectly, and an antibody 
targeting HMGB1 has been demonstrated to inhibit the angiogenesis in vitro and in vivo (29). HMGB1 was able to directly induce sprouting of endothelial cell spheroids in a collagen gel, and to stimulate endothelial cell proliferation, chemotaxis and repair of a wounded monolayer (30). HMGB1 exerts its angiogenetic effects via the RAGE pathway in tumor cells; it has been demonstrated to increase the expression of angiogenic growth factors, including VEGF, and to attract macrophages, which also produce a number of potent angiogenetic cytokines and growth factors (31). Furthermore, HMGB1 has been observed to attract endothelial progenitor cells (EPCs) and hematopoietic stem cells to sites of tissue injury and tumors to improve neovascularization mediated by RAGE, and antiRAGE antibodies were able to inhibit this process (32). The role of HMGB2 in angiogenesis has not yet been studied. We found that overexpression of HMGB1 and HMGB2 were connected with VEGF expression and MVD counts in BCa tissues, indicating that they have important roles in angiogenesis, partly through VEGF. However, the precise molecular mechanisms require further study.

In conclusion, our data revealed that the expression levels of HMGB1 and HMGB2 were highly increased in BCa, and that HMGB1/HMGB2 overexpression was significantly correlated with malignant tumor progression and angiogenesis. As potential diagnostic and therapeutic targets, further studies are required to understand the molecular mechanism of the involvement of HMGB1 and HMGB2 in BCa.

\section{Acknowledgements}

This study was supported by a grant from the Science and Technology Commission of Shanghai Municipality (Project No. 074119519).

\section{References}

1. Siegel R, Naishadham D and Jemal A: Cancer statistics, 2012. CA Cancer J Clin 62: 10-29, 2012.

2. Ghoneim MA, Abdel-Latif M, el-Mekresh M, et al: Radical cystectomy for carcinoma of the bladder: 2,720 consecutive cases 5 years later. J Urol 180: 121-127, 2008.

3. Vishnu P, Mathew J and Tan WW: Current therapeutic strategies for invasive and metastatic bladder cancer. Onco Targets Ther 4 97-113, 2011

4. van Beijnum JR, Buurman WA and Griffioen AW: Convergence and amplification of toll-like receptor (TLR) and receptor for advanced glycation end products (RAGE) signaling pathways via high mobility group B1 (HMGB1). Angiogenesis 11:91-99, 2008

5. Zhang J, McCauley MJ, Maher LJ III, et al: Mechanism of DNA flexibility enhancement by HMGB proteins. Nucleic Acids Res 37: 1107-1114, 2009.

6. Stros M, Polanská E, Struncová S and Pospísilová S: HMGB1 and HMGB2 proteins up-regulate cellular expression of human topoisomerase IIalpha. Nucleic Acids Res 37: 2070-2086, 2009.

7. Krynetskaia NF, Phadke MS, Jadhav SH and Krynetskiy EY: Chromatin-associated proteins HMGB1/2 and PDIA3 trigger cellular response to chemotherapy-induced DNA damage. Mol Cancer Ther 8: 864-872, 2009.

8. Balani P, Boulaire J, Zhao Y, et al: High mobility group box2 promoter-controlled suicide gene expression enables targeted glioblastoma treatment. Mol Ther 17: 1003-1011, 2009.

9. Naglova $\mathrm{H}$ and Bucova M: HMGB1 and its physiological and pathological roles. Bratisl Lek Listy 113: 163-171, 2012.

10. Franklin S, Chen H, Mitchell-Jordan S, et al: Quantitative analysis of the chromatin proteome in disease reveals remodeling principles and identifies high mobility group protein b2 as a regulator of hypertrophic growth. Mol Cell Proteomics 11: M111.014258, 2012.
11. Dong Xda E, Ito N, Lotze MT, et al: High mobility group box I (HMGB1) release from tumor cells after treatment: implications for development of targeted chemoimmunotherapy. J Immunother 30: 596-606, 2007.

12. Yao X, Zhao G, Yang H, et al: Overexpression of high-mobility group box 1 correlates with tumor progression and poor prognosis in human colorectal carcinoma. J Cancer Res Clin Oncol 136: 677-684, 2010

13. Shen X, Hong L, Sun H, et al: The expression of high-mobility group protein box 1 correlates with the progression of non-small cell lung cancer. Oncol Rep 22: 535-539, 2009.

14. Kostova N, Zlateva S, Ugrinova I and Pasheva E: The expression of HMGB1 protein and its receptor RAGE in human malignant tumors. Mol Cell Biochem 337: 251-258, 2010.

15. Sharma A, Ray R and Rajeswari MR: Overexpression of high mobility group (HMG) B1 and B2 proteins directly correlates with the progression of squamous cell carcinoma in skin. Cancer Invest 26: 843-851, 2008.

16. Gnanasekar M, Thirugnanam S and Ramaswamy K: Short hairpin RNA (shRNA) constructs targeting high mobility group box-1 (HMGB1) expression leads to inhibition of prostate cancer cell survival and apoptosis. Int J Oncol 34: 425-431, 2009.

17. Song B, Song WG, Li ZJ, et al: Effect of HMGB1 silencing on cell proliferation, invasion and apoptosis of MGC-803 gastric cancer cells. Cell Biochem Funct 30: 11-17, 2012

18. Rhodes DR, Yu J, Shanker K, et al: Large-scale meta-analysis of cancer microarray data identifies common transcriptional profiles of neoplastic transformation and progression. Proc Natl Acad Sci USA 101: 9309-9314, 2004.

19. Jiao Y, Wang HC and Fan SJ: Growth suppression and radiosensitivity increase by HMGB1 in breast cancer. Acta Pharmacol Sin 28: 1957-1967, 2007.

20. Tang D, Kang R, Zeh HJ III and Lotze MT: High-mobility group box 1 and cancer. Biochim Biophys Acta 1799: 131-140, 2010.

21. Sitohy B, Nagy J and Dvorak H: Anti-VEGF/VEGFR therapy for cancer: reassessing the target. Cancer Res 72: 1909-1914, 2012.

22. Ajili F, Kacem M, Tounsi H, et al: Prognostic impact of angiogenesis in nonmuscle invasive bladder cancer as defined by microvessel density after immunohistochemical staining for CD34. Ultrastruct Pathol 36: 336-342, 2012.

23. Deniz H, Karakök M, Yagci F and Güldür ME: Evaluation of relationship between HIF-1alpha immunoreactivity and stage, grade, angiogenic profile and proliferative index in bladder urothelial carcinomas. Int Urol Nephrol 42: 103-107, 2012.

24. Yang GL, Zhang LH, Bo JJ, et al: Increased expression of HMGB1 is associated with poor prognosis in human bladder cancer. J Surg Oncol 106: 57-61, 2012.

25. Tang D, Kang R, Cheh CW, et al: HMGB1 release and redox regulates autophagy and apoptosis in cancer cells. Oncogene 29: 5299-5310, 2010.

26. Todorova $\mathrm{J}$ and Pasheva E: High mobility group B1 protein interacts with its receptor RAGE in tumor cells but not in normal tissues. Oncol Lett 3: 214-218, 2012.

27. Pusterla T, de Marchis F, Palumbo R and Bianchi ME: High mobility group B2 is secreted by myeloid cells and has mitogenic and chemoattractant activities similar to high mobility group B1. Autoimmunity 42: 308-310, 2009.

28. Le XF, Lammayot A, Gold D, et al: Genes affecting the cell cycle, growth, maintenance, and drug sensitivity are preferentially regulated by anti-HER 2 antibody through phosphatidylinositol 3-kinase-AKT signaling. J Biol Chem 280: 2092-2104, 2005.

29. van Beijnum JR, Nowak-Sliwinska P, van den Boezem E, et al: Tumor angiogenesis is enforced by autocrine regulation of highmobility group box 1. Oncogene: Mar 5, 2012 (Epub ahead of print). doi: $10.1038 /$ onc.2012.49.

30. Sachdev U, Cui X, Hong G, et al: High mobility group box 1 promotes endothelial cell angiogenic behavior in vitro and improves muscle perfusion in vivo in response to ischemic injury. J Vasc Surg 55: 180-191, 2012.

31. Urquidi V, Goodison S, Kim J, et al: Vascular endothelial growth factor, carbonic anhydrase 9 , and angiogenin as urinary biomarkers for bladder cancer detection. Urology 79: 1185.e1-6, 2012.

32. Chavakis E, Hain A, Vinci M, et al: High-mobility group box 1 activates integrin-dependent homing of endothelial progenitor cells. Circ Res 100: 204-212, 2007. 